\title{
Radial sign-changing solutions to biharmonic nonlinear Schrödinger equations
}

\section{Marcos TO Pimenta*}

\section{"Correspondence:}

pimenta@fct.unesp.br

Departamento de Matemática e

Computação, Faculdade de Ciências

e Tecnologia, Universidade Estadual

Paulista - Unesp, Presidente

Prudente, SP 19060-900, Brazil

\begin{abstract}
In this work we obtain three radial solutions of a biharmonic stationary Schrödinger equation, one being positive, one negative, and one sign changing. The dual decomposition method is used to split the natural second-order Sobolev space considered in order to apply the appropriate variational approach.
\end{abstract}

MSC: $35 J 60 ; 35 J 35$

Keywords: variational methods; biharmonic equations; nodal solutions

\section{Introduction}

In the last two decades, the stationary nonlinear Schrödinger equation, given by

$$
\left\{\begin{array}{l}
-\epsilon^{2} \Delta u+V(x) u=f(x, u) \quad \text { in } \mathbb{R}^{N}, \\
u \in H^{1}\left(\mathbb{R}^{N}\right),
\end{array}\right.
$$

has been widely studied by several authors in works dealing with the existence, the multiplicity, and the concentration of solutions when the parameter $\epsilon \rightarrow 0$. In order to highlight some of the most influential works about this subject, we could quote the pioneering work of Floer and Weinstein [1], in which they used the Lyapunov-Schmidt reduction to obtain positive solutions exhibiting the concentration behavior for the unidimensional case. In [2], Rabinowitz used a variational approach to obtain positive solutions of (1.1) under global hypotheses on the potential $V$. Later, Wang in [3] proved that the solutions obtained by Rabinowitz concentrate around the global infimum of the potential. In the celebrated paper [4], del Pino and Felmer used the so called penalization technique to prove the same kind of concentration behavior of solutions of (1.1), considering the potential $V$ under a local version of the Rabinowitz condition.

In all of the above mentioned works, the authors worked with potentials $V$ bounded away from zero. Stationary NLS problems with vanishing potentials were treated for instance by Bonheure et al. in [5, 6], where the authors obtained concentration of positive solutions around global minimum points of an auxiliary function and even around some lower dimensional spheres in $\mathbb{R}^{N}$.

In spite of this amount of results treating the second-order case, just few works can be found dealing with similar questions involving the fourth-order equation

$$
\left\{\begin{array}{l}
\Delta^{2} u+V(x) u=f(x, u) \quad \text { in } \mathbb{R}^{N}, \\
u \in H^{2}\left(\mathbb{R}^{N}\right) .
\end{array}\right.
$$

(0) 2015 Pimenta; licensee Springer. This is an Open Access article distributed under the terms of the Creative Commons Attribution License (http://creativecommons.org/licenses/by/4.0), which permits unrestricted use, distribution, and reproduction in any medium, provided the original work is properly credited. 
In $[7,8]$, the author and Soares proved the existence of a concentrating sequence of solutions of the singular perturbed version of (1.2), considering, respectively, a global and a local condition on the potential $V$, and a subcritical power-type nonlinearity $f$. The main arguments of these works were strongly inspired by Rabinowitz [2], Wang [3] and del Pino and Felmer [4], in which other arguments to overcome the lack of a maximum principle of the biharmonic operator were required. In [9] the author and Figueiredo, using LjusternikSchnirelmann theory, deal with the same problem as in [8], obtaining multiple solutions exhibiting the concentration phenomenon.

Another relevant question about both problems (1.1) and (1.2) is the existence of signchanging solutions, sometimes called nodal solutions. For the second-order problem there are several papers dealing with this subject. In a pioneering work [10], Castro et al. have obtained three solutions, including a nodal one, for a second-order problem in a bounded domain and with Dirichlet boundary conditions. In [11,12], Alves and Soares use the penalization technique to get nodal solutions concentrating around extremal points of the potential $V$. In this approach, they use arguments based on the minimization of the energy functional in some Nehari sets, considering $u^{+}=\max \{u, 0\}$ and $u^{-}=\min \{u, 0\}$, respectively, the positive and negative parts of a function $u \in H^{1}\left(\mathbb{R}^{N}\right)$. At first sight, one could think that these arguments are trivially adaptable to the fourth-order case, however, as long as in $H^{1}\left(\mathbb{R}^{N}\right)$ the decomposition $u=u^{+}+u^{-}$is trivially allowed; in $H^{2}\left(\mathbb{R}^{N}\right)$ this factorization is no longer to be available.

In this work, we consider the problem (1.2) where $N \geq 5$, and $f$ and $V$ satisfy the following assumption set:

$\left(\mathrm{V}_{1}\right) \quad 0<V_{0}:=\inf _{\mathbb{R}^{N}} V$ and $V(x)=V(|x|)$, for all $x \in \mathbb{R}^{N}$;

$\left(\mathrm{V}_{2}\right)$ there exist $\Omega \subset \mathbb{R}^{N}, 0<V_{0}:=\inf _{\mathbb{R}^{N}} V$, and $V(x)=V(|x|)$, for all $x \in \mathbb{R}^{N}$;

$\left(\mathrm{f}_{1}\right) \quad f: \mathbb{R}^{N} \times \mathbb{R} \rightarrow \mathbb{R}$ is a Carathéodory function;

$\left(\mathrm{f}_{2}\right) \quad f(x, s)=o(|s|)$ as $s \rightarrow 0$ a.e. in $\mathbb{R}^{N}$;

$\left(\mathrm{f}_{3}\right)$ there are constants $c_{1}, c_{2}>0$, and $0<p<2 *-2$, where $2_{*}=2 N /(N-4)$, such that

$$
|f(x, s)-f(x, t)| \leq\left(c_{1}+c_{2}\left(|s|^{p}+|t|^{p}\right)\right)|s-t|, \quad \text { for a.e. } x \in \mathbb{R}^{N} \text { and } s, t \in \mathbb{R} ;
$$

(f $\left.f_{4}\right) \quad \lim _{|s| \rightarrow \infty} \frac{F(x, s)}{s^{2}}=+\infty$ a.e. in $\mathbb{R}^{N}$, where $F(x, s)=\int_{0}^{s} f(x, t) d t$;

$\left(f_{5}\right) \quad \frac{f(x, s)}{s}$ is nondecreasing for $s>0$ and nonincreasing for $s<0$, for a.e. $x \in \mathbb{R}^{N}$.

Remark 1.1 Note that $\left(f_{5}\right)$ implies that $f(x, s)$ is nondecreasing in $\mathbb{R}$ and that $(x, s) \mapsto$ $f(x, s) s-2 F(x, s)$ is also nondecreasing for $s>0$ and nonincreasing for $s<0$, for a.e. $x \in \mathbb{R}^{N}$.

Remark 1.2 An example of nonlinearity satisfying $\left(\mathrm{f}_{1}\right)-\left(\mathrm{f}_{5}\right)$ is $f(x, s)=\sum_{i=1}^{k} a_{i}(x)|s|^{p_{i}} s$, where $a_{i} \in L^{\infty}\left(\mathbb{R}^{N}\right), a_{i} \geq 0$ in a positive measure set of $\mathbb{R}^{N}$, and $0<p_{i}<2_{*}-2$, for all $i \in\{1, \ldots, k\}$.

In order to overcome the lack of the decomposition of $H^{2}\left(\mathbb{R}^{N}\right)$ in terms of positive and negative parts of their functions, we use an alternative method developed by Moreau in [13] called the dual cones decomposition method, which consists in splitting a function $u$ in a Hilbert space $H$ as $u=u_{1}+u_{2}$, in such a way that $u \in \mathcal{K}$ and $u_{2} \in \mathcal{K}^{*}$, where $\mathcal{K}$ is a cone in $H$ and $\mathcal{K}^{*}$ is called its dual cone. Considering $H=H^{2}\left(\mathbb{R}^{N}\right)$ and $\mathcal{K}=\left\{u \in H^{2}\left(\mathbb{R}^{N}\right) ; u \geq 0\right\}$, if it is possible to prove that $\mathcal{K}^{*} \subset-\mathcal{K}$, then we have a decomposition of a function $u \in$ 
$H^{2}\left(\mathbb{R}^{N}\right)$ in terms of a non-negative and a non-positive function such that many times we can substitute the trivial decomposition not available in our case. Our approach follows closely the work of Weth [14], in which the author takes advantage of the Moreau method to obtain signed and sign-changing solutions of the problem

$$
\Delta^{2} u=f(x, u) \text { in } \Omega
$$

under Dirichlet or Navier boundary conditions in a bounded domain $\Omega$. It is worth pointing out that to prove that $\mathcal{K}^{*} \subset-\mathcal{K}$, it is necessary that we have some kind of a maximum principle to $\Delta^{2}$. In fact, this is the main reason why this method becomes so restrictive when dealing with fourth-order problems. In [14], Weth uses the fact that under Navier boundary conditions, one can use twice the strong maximum principle to $-\Delta$ in $\Omega$, in order to obtain a version of this result to $\Delta^{2}$. Considering Dirichlet boundary conditions, the same is true at least to some domains like balls and limaçons. In this sense, our first contribution is overcoming the lack of a maximum principle of $\Delta^{2}$ in $\mathbb{R}^{N}$ by using some arguments of Chabrowski and Yang in [15] to prove that there exist positive solutions of a linear version of (1.2), which in particular implies that $\mathcal{K}^{*} \subset-\mathcal{K}$ (see Lemma 2.10).

Another difficulty that deserves to be highlighted is the lack of compactness, since the problem is in $\mathbb{R}^{N}$. In order to overcome this difficulty, we consider the problem restricted to $H_{\text {rad }}^{2}\left(\mathbb{R}^{N}\right)$ consisting in the radial functions belonging to $H^{2}\left(\mathbb{R}^{N}\right)$. This is interesting because of a version of the Strauss lemma as regards higher-order Sobolev spaces proved by Ebihara and Schonbek in [16]. At the end, once critical points of the restricted energy functional are at hand we obtain critical points of the functional using the principle of symmetric criticality of Palais.

Huang and Liu, in very recent papers $[17,18]$, have applied similar arguments to prove the existence of sign-changing solutions to, respectively, a biharmonic and a $p$-biharmonic problem. In the first one the authors have studied a biharmonic problem with an asymptotic linear nonlinearity and in the second, a $p$-biharmonic problem with a Hardy type potential.

Our main result is the following.

Theorem 1.3 Assume that conditions $\left(\mathrm{V}_{1}\right)$ and $\left(\mathrm{f}_{1}\right)-\left(\mathrm{f}_{5}\right)$ hold. Then there exist at least one positive, one negative and one nodal classical radial solution of (1.2).

The proof involves variational arguments consisting in searching for critical points of the energy functional, looking for stationary points of a Cauchy problem in Banach spaces. In this sense, some results of Liu and Sun [19] about the invariance of some sets will be necessary.

Finally, we note that the nonlinearity $f$ does not satisfy the well-known AmbrosettiRabinowitz super-linearity condition, given by

(AR) there exists $\mu>2$ such that $0<\mu F(x, s) \leq f(x, s) s$, for all $s \neq 0$ and a.e. in $\mathbb{R}^{N}$. Instead of this condition, in order to increase the range of admissible nonlinearities, we consider the weaker assumption $\left(\mathrm{f}_{4}\right)$. This requires some arguments of Miyagaki and Souto in [20] to prove the boundedness of a certain sequence.

When proving Theorem 1.3 our main contribution is in providing a result on the existence of nodal solutions to the BNLS equation, which seems to be difficult to obtain 
by other methods like Nehari analysis. In fact the real difficulty here is in overcoming the lack of the strong maximum principle in applying a method which works very well in those situations where this principle is available.

In the first section we describe the variational framework. In the second one we introduce the Cauchy problem and prove the invariance of some sets. The last section is left for the proof of the main result.

To save notation in all of this paper we denote $\int_{\mathbb{R}^{N}} g d x$ just by $\int g$. The norm $\|\cdot\|_{L^{p}\left(\mathbb{R}^{N}\right)}$ will be simple denoted by $\|\cdot\|_{p}$.

\section{The variational framework}

As mentioned in the introduction, in order to overcome the lack of compactness, let us consider $H=H_{\text {rad }}^{2}\left(\mathbb{R}^{N}\right)$ which is a Hilbert space when endowed with the following inner product:

$$
\langle u, v\rangle=\int(\Delta u \Delta v+V(x) u v)
$$

which gives rise to the following norm:

$$
\|u\|=\left(\int\left(|\Delta u|^{2}+V(x) u^{2}\right)\right)^{\frac{1}{2}} .
$$

By $\left(\mathrm{V}_{1}\right)$, it follows easily that $\|\cdot\|$ is equivalent to the usual norm in $H^{2}\left(\mathbb{R}^{N}\right)$.

Before introducing the energy functional associated to (1.2), let us remember some results proved by Ebihara and Schonbek in [16] that will be used along this text.

Lemma 2.1 (Corollary 2 in [16]) The following embeddings are compact:

$$
\begin{aligned}
& H_{\text {rad }}^{2}\left(\mathbb{R}^{N}\right) \hookrightarrow L^{q}\left(\mathbb{R}^{N}\right), \quad \text { for all } 2<q<2_{*} \text { if } N \geq 5, \\
& H_{\text {rad }}^{2}\left(\mathbb{R}^{N}\right) \hookrightarrow L^{q}\left(\mathbb{R}^{N}\right), \quad \text { for all } 2<q \text { if } N=1,2,3,4 .
\end{aligned}
$$

Lemma 2.2 (Theorem 2.1 in [16]) $H_{\text {rad }}^{2}\left(\mathbb{R}^{N}\right)$ is not compactly embedded into $L^{2}\left(\mathbb{R}^{N}\right)$.

Let us consider the restriction to $H$ of the energy functional whose Euler-Lagrange equation is (1.2), $I: H \rightarrow \mathbb{R}$, given by

$$
I(u)=\frac{1}{2} \int\left(|\Delta u|^{2}+V(x) u^{2}\right)-\int F(x, u)=\frac{1}{2}\|u\|^{2}-\int F(x, u) .
$$

Note that by $\left(\mathrm{f}_{3}\right)$ and Sobolev embeddings, $I$ is well defined.

Since for each $u \in H, v \mapsto \int f(x, u) v$ is a continuous linear functional in $H$, it is well defined $A(u) \in H$ such that

$$
\langle A(u), v\rangle=\int f(x, u) v, \quad \text { for all } v \in H .
$$

The following result states some interesting properties of the operator $A: H \rightarrow H$ defined above. 
Lemma 2.3 $A$ is a compact, locally Lipschitz operator, such that $A(u)=\nabla \Psi$ where $\Psi$ : $H \rightarrow \mathbb{R}$ is given by $\Psi(u)=\int F(x, u)$. Moreover, for each $\epsilon>0$, there exists $A(\epsilon)>0$ such that

$$
|\langle A(u), v\rangle| \leq\left(\epsilon\|u\|+A(\epsilon)\|u\|^{p+1}\right)\|v\|, \quad \text { for all } u, v \in H,
$$

where $p$ is given in $\left(\mathrm{f}_{3}\right)$.

Proof Let us prove the estimate. Note that from $\left(\mathrm{f}_{2}\right)$ and $\left(\mathrm{f}_{3}\right)$ it follows that for each $\epsilon<0$, there exists $A(\epsilon)>0$ such that

$$
|f(x, s)| \leq \epsilon|s|+A(\epsilon)|s|^{p+1}, \quad \text { for a.e. } x \in \mathbb{R}^{N} \text { and for all } s \in \mathbb{R} \text {. }
$$

Using Hölder with the conjugated exponents $\frac{2_{*}}{p+1}$ and $\frac{2_{*}}{2_{*}-(p+1)}$, we have

$$
\begin{aligned}
|\langle A(u), v\rangle| & \leq \int\left(\epsilon|u|+A(\epsilon)|u|^{p+1}\right)|v| \\
& \leq \epsilon\|u\|\|v\|+A(\epsilon)\left\|u^{p+1}\right\|\|v\| .
\end{aligned}
$$

Just by definition and Lebesgue dominated convergence theorem, it follows that $A=\nabla \Psi$, where $\Psi(u)=\int F(u)$. On the other hand, $\left(\mathrm{f}_{2}\right)$ implies that $A$ is a locally Lipschitz operator.

What is left to show is that $A$ is a compact operator. Although this follows by straightforward calculations, we describe all the details, since this was the reason why we had to consider the space $H_{\text {rad }}^{2}\left(\mathbb{R}^{N}\right)$ rather than $H^{2}\left(\mathbb{R}^{N}\right)$.

Let $\left(u_{n}\right) \subset H_{\text {rad }}^{2}\left(\mathbb{R}^{N}\right)$ be a bounded sequence. Along a subsequence, we have

$$
\begin{array}{ll}
u_{n} \rightarrow u & \text { in } H_{\mathrm{rad}}^{2}\left(\mathbb{R}^{N}\right), \\
u_{n} \rightarrow u & \text { in } L^{q}\left(\mathbb{R}^{N}\right), \text { for } 2<q<2 N /(N-4) .
\end{array}
$$

For each $v \in C_{0}^{\infty}\left(\mathbb{R}^{N}\right)$, it follows by $\left(\mathrm{f}_{3}\right)$ and the Hölder inequality with $2+\epsilon$ and $(2+\epsilon)^{\prime}$, for small enough $\epsilon>0$, that

$$
\begin{aligned}
& \left.|| A\left(u_{n}\right)-A(u), v\right\rangle \mid \\
& \quad \leq \int\left|f\left(x, u_{n}\right)-f(x, u)\right||v| \\
& \quad \leq \int_{\operatorname{supp}(v)} c_{1}\left|u_{n}-u\right||v|+\int_{\operatorname{supp}(v)} c_{2}\left(\left|u_{n}\right|^{p}+|u|^{p}\right)\left|u_{n}-u\right||v| \\
& \quad \leq C(v)\left\|u_{n}-u\right\|_{2+\epsilon}+C(v)\left(\int_{\operatorname{supp}(v)}\left|u_{n}\right|^{p}\left|u_{n}-u\right|+\int_{\operatorname{supp}(v)}|u|^{p}\left|u_{n}-u\right|\right) .
\end{aligned}
$$

By taking $2<r<\min \left\{2,2_{*} / p\right\}$, using the Hölder inequality with $r$ and $r^{\prime}$ and by (2.1), we obtain

$$
\begin{aligned}
\left|\left\langle A\left(u_{n}\right)-A(u), v\right\rangle\right| & \leq C(v) o_{n}(1)+C(v)\left\|u_{n}\right\|_{p, r}^{p}\left\|u_{n}-u\right\|_{r^{\prime}}+C(v)\|u\|_{p, r}^{p}\left\|u_{n}-u\right\|_{r^{\prime}} \\
& =o_{n}(1) .
\end{aligned}
$$


Therefore, for all $v \in C_{0}^{\infty}\left(\mathbb{R}^{N}\right)$ it follows that

$$
\lim _{n \rightarrow \infty}\left\langle A\left(u_{n}\right)-A(u), v\right\rangle=0 .
$$

Since $C_{0}^{\infty}\left(\mathbb{R}^{N}\right)$ is dense in $H_{\text {rad }}^{2}\left(\mathbb{R}^{N}\right)$, it follows that (2.2) holds for all $v \in H_{\text {rad }}^{2}\left(\mathbb{R}^{N}\right)$, and this implies that

$$
\lim _{n \rightarrow \infty} A\left(u_{n}\right)=A(u) .
$$

It is straightforward to prove that critical points of $I$ correspond to fixed points of $A$. Let us consider the following Cauchy problem in the Hilbert space $H$ :

$$
\left\{\begin{array}{l}
\frac{\partial}{\partial t} \varphi(t, u)=-\nabla I(\varphi(t, u))=A(\varphi(t, u))-\varphi(t, u), \\
\varphi(0, u)=u
\end{array}\right.
$$

where $\varphi: \mathcal{G} \rightarrow H$ and $\mathcal{G}=\{(t, u) \in \mathbb{R} \times H ; u \in H$ and $t \in[0, T(u))\}$ and $[0, T(u))$ is the maximal interval of the existence of the trajectory $t \mapsto \varphi(t, u)$. Note that since $A$ is a Lipschitz continuous operator, the flow $\varphi$ is well defined.

The following is a key point in our approach.

Proposition 2.4 If for some $u \in H,\{I(\varphi(t, u)) ; 0 \leq t<T(u)\}$ is bounded from below, then:

(i) $T(u)=\infty$,

(ii) there exists $t_{n} \rightarrow \infty$ such that $\left\{\varphi\left(t_{n}, u\right), n \in \mathbb{N}\right\}$ is bounded in $H$ and the $\omega$-limit set of $u$,

$$
\omega(u)=\bigcap_{0 \leq t<\infty} \overline{\bigcup_{t \leq s<\infty} \varphi(s, u)}
$$

is a non-empty set formed by critical points of $I$.

Proof (i) Note that

$$
\begin{aligned}
\|\varphi(t, u)-\varphi(s, u)\| & \leq \int_{s}^{t}\|\nabla I(\varphi(\tau, u))\| d \tau \\
& \leq \sqrt{t-s}\left(\int_{s}^{t}\|\nabla I(\varphi(\tau, u))\|^{2} d \tau\right)^{\frac{1}{2}} \\
& =\sqrt{t-s}(I(\varphi(s, u))-I(\varphi(t, u)))^{\frac{1}{2}},
\end{aligned}
$$

where we have used Hölder and (2.3). Suppose the assertion of the item is false. Then by the last estimate, the trajectory $\{\varphi(t, u) ; t \in[0, T(u))\}$ would be bounded, which implies that $T(u)=\infty$, which gives rise to a contradiction.

(ii) First of all, let us note that there exists a sequence $t_{n} \rightarrow \infty$ such that $\left\|\nabla I\left(\varphi\left(t_{n}, u\right)\right)\right\| \rightarrow$ 0 , as $n \rightarrow \infty$. This follows just by noting that

$$
\begin{aligned}
\int_{0}^{\infty}\|\nabla I(\varphi(\tau, u))\|^{2} d \tau & =\lim _{t \rightarrow \infty} \int_{0}^{t}\|\nabla I(\varphi(\tau, u))\|^{2} d \tau \\
& =\lim _{t \rightarrow \infty}|I(\varphi(t, u))-I(u)|<\infty .
\end{aligned}
$$


We claim that $\left\{\varphi\left(t_{n}, u\right)\right\}_{n \in \mathbb{N}}$ is uniformly bounded in $H$ with respect to $n \in \mathbb{N}$.

Suppose, contrary to our claim, that $\left\|\varphi\left(t_{n}, u\right)\right\| \rightarrow \infty$ as $n \rightarrow \infty$. Let us define

$$
w_{n}=\frac{\varphi\left(t_{n}, u\right)}{\left\|\varphi\left(t_{n}, u\right)\right\|}
$$

Since $\left(w_{n}\right)$ is a bounded sequence in $H$, it follows that there exists $w \in H$ such that $w_{n} \rightarrow w$ in $H$, up to a subsequence. Then Lemma 2.1 implies that $w_{n} \rightarrow w$ in $L^{q}\left(\mathbb{R}^{N}\right), 2<q<2_{*}$, and also $w_{n} \rightarrow w$ a.e. in $\mathbb{R}^{N}$.

In order to prove that $w=0$, let us consider $\Gamma=\left\{x \in \mathbb{R}^{N} ; w(x) \neq 0\right\}$, and prove that $\Gamma$ has zero Lebesgue measure. For all $x \in \Gamma$, we have $\lim _{n \rightarrow \infty} \varphi\left(t_{n}, u\right)(x)=\infty$. By $\left(f_{4}\right)$, for each $M>0$, there exists $r>0$ such that

$$
F(x, s) \geq M s^{2}, \quad \text { for all } s \geq r \text { and for a.e. } x \in \mathbb{R}^{N} .
$$

Since $\left\{I\left(\varphi\left(t_{n}, u\right)\right)\right\}_{n \rightarrow \infty}$ is bounded from below,

$$
\begin{aligned}
\frac{1}{2}+o_{n}(1) & \geq \int \frac{F\left(x, \varphi\left(t_{n}, u\right)\right)}{\left\|\varphi\left(t_{n}, u\right)\right\|^{2}} \\
& \geq \int_{\left\{\left|\varphi\left(t_{n}, u\right)\right|>r\right\} \cap \Gamma} \frac{F\left(x, \varphi\left(t_{n}, u\right)\right)}{\varphi\left(t_{n}, u\right)^{2}} w_{n}^{2} \\
& \geq M \int_{\left\{\left|\varphi\left(t_{n}, u\right)\right|>r\right\} \cap \Gamma} w_{n}^{2} .
\end{aligned}
$$

Observing that $\varphi\left(t_{n}, u\right)(x) \rightarrow+\infty$ as $n \rightarrow \infty$ for all $x \in \Gamma$ and by Fatou's lemma, it follows that

$$
\frac{1}{2} \geq M \int_{\Gamma} w^{2}
$$

which is a contradiction since $\int_{\Gamma} w^{2}>0$ and $M$ is arbitrary. Hence, $|\Gamma|=0$.

Note that the function $t \mapsto I\left(t \varphi\left(t_{n}, u\right)\right)$ is smooth in $(0,1)$. Let $s_{n} \in[0,1]$ such that

$$
I\left(s_{n} \varphi\left(t_{n}, u\right)\right)=\max _{t \in[0,1]} I\left(t \varphi\left(t_{n}, u\right)\right) .
$$

For each $R>0$, and for all $n$ large enough,

$$
\begin{aligned}
I\left(s_{n} \varphi\left(t_{n}, u\right)\right) & \geq I\left(\frac{R}{\left\|\varphi\left(t_{n}, u\right)\right\|} \varphi\left(t_{n}, u\right)\right) \\
& =\frac{R^{2}}{2}-\int F\left(x, R w_{n}\right)=\frac{R^{2}}{2}+o_{n}(1) .
\end{aligned}
$$

Hence $\lim _{n \rightarrow \infty} I\left(s_{n} \varphi\left(t_{n}, u\right)\right)=+\infty$, which implies that $s_{n} \in(0,1)$. Then, for $n$ large enough $I^{\prime}\left(s_{n} \varphi\left(t_{n}, u\right)\right) s_{n} \varphi\left(t_{n}, u\right)=0$. By $\left(f_{4}\right)$, for all $t \in[0,1]$ we have

$$
\begin{aligned}
2 I\left(t \varphi\left(t_{n}, u\right)\right) & \leq 2 I\left(s_{n} \varphi\left(t_{n}, u\right)\right)-I^{\prime}\left(s_{n} \varphi\left(t_{n}, u\right)\right) s_{n} \varphi\left(t_{n}, u\right) \\
& =\int\left(f\left(x, s_{n} \varphi\left(t_{n}, u\right)\right) s_{n} \varphi\left(t_{n}, u\right)-2 F\left(x, s_{n} \varphi\left(t_{n}, u\right)\right)\right)
\end{aligned}
$$




$$
\begin{aligned}
& \leq \int\left(f\left(x, \varphi\left(t_{n}, u\right)\right) \varphi\left(t_{n}, u\right)-2 F\left(x, \varphi\left(t_{n}, u\right)\right)\right) \\
& =2 I\left(\varphi\left(t_{n}, u\right)\right)+o_{n}(1) \leq C_{1} .
\end{aligned}
$$

For a given $R_{0}>0$, for $n$ large enough, it follows that $\frac{R_{0}}{\left\|\varphi\left(t_{n}, u\right)\right\|}<1$. Then

$$
2 I\left(R_{0} w_{n}\right)=2 I\left(\frac{R_{0}}{\left\|\varphi\left(t_{n}, u\right)\right\|} \varphi\left(t_{n}, u\right)\right) \leq C_{1}
$$

On the other hand, for all $R_{0}>0$,

$$
2 I\left(R_{0} w_{n}\right)=R_{0}^{2}-2 \int F\left(x, R_{0} w_{n}\right)=R_{0}^{2}+o_{n}(1) .
$$

Since (2.4) contradicts (2.5), we find that $\left\{\varphi\left(t_{n}, u\right)\right\}_{n \in \mathbb{N}}$ is uniformly bounded in $H$ with respect to $n \in \mathbb{N}$ and the claim is proved.

In order to show that $\omega(u) \neq \emptyset$, let us consider the bounded sequence $\left\{\varphi\left(t_{n}, u\right)\right\}$. Since $A$ is compact, there exists $u_{0} \in H$ such that $A\left(\varphi\left(t_{n}, u\right)\right) \rightarrow u_{0}$ along a subsequence. Hence

$$
\begin{aligned}
0 & =\lim _{n \rightarrow \infty}\left\|\nabla I\left(\varphi\left(t_{n}, u\right)\right)\right\| \\
& =\lim _{n \rightarrow \infty}\left(\varphi\left(t_{n}, u\right)-A\left(\varphi\left(t_{n}, u\right)\right)\right) \\
& =\lim _{n \rightarrow \infty}\left(\varphi\left(t_{n}, u\right)-u_{0}\right) .
\end{aligned}
$$

Then $\lim _{n \rightarrow \infty} \varphi\left(t_{n}, u\right)=u_{0}$ and $u_{0} \in \omega(u)$.

Concerning the proof that every point in $\omega(u)$ is a critical point of $I$, note that $I(\varphi(t, u)) \rightarrow d$ as $t \rightarrow+\infty$. Then, if $v \in \omega(u)$, there exists $t_{n} \rightarrow \infty$ such that $\varphi\left(t_{n}, u\right) \rightarrow v$ in $H$ as $n \rightarrow \infty$. Hence

$$
I(\varphi(t, v))=\lim _{n \rightarrow \infty} I\left(\varphi\left(t, \varphi\left(t_{n}, u\right)\right)\right)=\lim _{n \rightarrow \infty} I\left(\varphi\left(t+t_{n}, u\right)\right)=d,
$$

for all $t \geq 0$. Then, for all $t \geq 0$,

$$
0=\frac{\partial}{\partial t} I(\varphi(t, v))=\|\nabla I(\varphi(t, v))\|^{2}
$$

which implies that $\nabla I(v)=0$ and $v$ is a critical point of $I$.

Definition 2.5 We call $D \subset H$ a positive invariant set if $\varphi(t, u) \in D$ for all $t \in[0, T(u))$ and $u \in D$. If $D$ is a positive invariant set we define its absorption domain by

$$
\mathcal{A}(D)=\left\{u \in H ; \exists t_{0} \in[0, T(u)) \text { such that } \varphi\left(t_{0}, u\right) \in D\right\} .
$$

Let us define the following set:

$$
\mathcal{A}_{0}=\{u \in H ; T(u)=\infty \text { and } \varphi(t, u) \rightarrow 0 \text { as } t \rightarrow \infty\} .
$$

Lemma 2.6 $\mathcal{A}_{0}$ is an open subset of $H$ and there exists $r>0$ such that $B_{r}(0) \subset \mathcal{A}_{0}$. 
Proof Let $0<\epsilon<1$ and $A(\epsilon)$ given in Lemma 2.3. Let us consider $\alpha_{0}=\left(\frac{1-\epsilon}{2 A(\epsilon)}\right)^{\frac{1}{p}}$. For each $u \in \overline{B_{\alpha_{0}}(0)}$, by Lemma 2.3 we have

$$
\begin{aligned}
\Psi(u) & =\int_{0}^{1} \frac{\partial}{\partial t} \Psi(t u) d t \\
& =\int_{0}^{1}\langle A(t u), u\rangle d t \\
& \leq \int_{0}^{1}\left(\epsilon\|t u\|+A(\epsilon)\|t u\|^{p+1}\right)\|u\| d t \\
& \leq \int_{0}^{1} t\|u\|^{2}\left(\epsilon+A(\epsilon)\|u\|^{p}\right) d t \\
& =\frac{\|u\|^{2}}{2}\left(\epsilon+A(\epsilon)\|u\|^{p}\right) \\
& \leq \frac{\|u\|^{2}}{2}\left(\epsilon+A(\epsilon) \alpha_{0}^{p}\right) \\
& =\frac{\epsilon+1}{4}\|u\|^{2} .
\end{aligned}
$$

Then

$$
I(u)=\frac{1}{2}\|u\|^{2}-\Psi(u) \geq\left(\frac{1}{2}-\frac{\epsilon+1}{4}\right)\|u\|^{2} \geq 0,
$$

since $\epsilon \in(0,1)$.

Moreover, for all $u \in \partial B_{\alpha_{0}}(0)$,

$$
I(u) \geq\left(\frac{1}{2}-\frac{\epsilon+1}{4}\right) \alpha_{0}^{2}=: \beta_{0}>0
$$

By continuity, there exists $r \in\left(0, \alpha_{0}\right)$ such that

$$
I(u)<\beta_{0}, \quad \text { for all } u \in B_{r}(0) .
$$

Since the energy does not grow along any trajectory, if $u \in B_{r}(0)$, then $I(\varphi(t, u))<\beta_{0}$, for all $t \in[0, T(u))$. Then, by (2.6), $\varphi(t, u) \in B_{\alpha_{0}}(0)$ for all $t \in[0, T(u))$. Hence $I(\varphi(t, u)) \geq 0$ for all $t \in[0, T(u))$, which implies that $T(u)=\infty$. Moreover, $\omega(u) \subset B_{\alpha_{0}}(0)$ is a compact non-empty set formed of critical points of $I$. On the other hand, if $v \in B_{\alpha_{0}}(0)$ is a critical point of $I$, then

$$
\|v\|^{2}=\langle A(v), v\rangle \leq\|v\|^{2}\left(\epsilon+A(\epsilon)\|v\|^{p}\right) \leq\|v\|^{2}\left(\epsilon+A(\epsilon) \alpha_{0}^{p}\right)=\frac{\epsilon+1}{2}\|v\|^{2},
$$

which only is possible in the case where $v=0$. Therefore, $\omega(u)=\{0\}$ for all $u \in B_{r}(0)$ and then $B_{r}(0) \subset \mathcal{A}_{0}$, which implies that $\mathcal{A}_{0}=\mathcal{A}\left(B_{r}(0)\right)$. Since $B_{r}(0)$ is an open set, then so is $\mathcal{A}\left(B_{r}(0)\right)$.

The following is a key result in our argument and in particular implies that $\partial \mathcal{A}_{0}$ is a great place to look for nontrivial critical points of $I$. 
Proposition $2.7 \partial \mathcal{A}_{0}$ is a closed positively invariant set of $H$ and $\inf _{u \in \partial \mathcal{A}_{0}} I(u) \geq 0$. In particular, for all $u \in \partial \mathcal{A}_{0}, \omega(u)$ is a non-empty set consisting in nontrivial critical points of $I$.

The proof of the positively invariance can be found in [19] while the other results are straightforward to see.

Although $\partial \mathcal{A}_{0}$ is a great set to look for nontrivial critical points of $I$, once found, nothing can be said about its signal. Let us introduce the concept of dual cone and state the dual cone decomposition theorem, which is given by Moreau in [13].

Definition 2.8 Given a cone $\mathcal{K}$ in a Hilbert space $H$, its dual cone is defined by

$$
\mathcal{K}^{*}=\{v \in H ;\langle u, v\rangle \leq 0, \forall u \in \mathcal{K}\}
$$

Theorem 2.9 Let $\mathcal{K} \subset H$ a closed convex cone. Then for all $x \in H$, there exist $y \in \mathcal{K}$ and $z \in \mathcal{K}^{*}$ such that

$$
x=y+z \quad \text { and } \quad\langle y, z\rangle=0 .
$$

Let us define the following cones and afterwards prove some invariance properties of them.

Let

$$
\begin{aligned}
& \mathcal{K}=\left\{u \in H ; u \geq 0 \text { a.e. in } \mathbb{R}^{N}\right\}, \\
& -\mathcal{K}=\left\{u \in H ; u \leq 0 \text { a.e. in } \mathbb{R}^{N}\right\},
\end{aligned}
$$

which are closed convex cones.

Let us denote by $P$ and $Q$ the orthogonal projections of $H$ in $\mathcal{K}$ and $-\mathcal{K}$, respectively. Denoting by $P^{*}=\mathrm{Id}-P$ and $Q^{*}=\operatorname{Id}-Q$, note that

$$
\left\langle P u, P^{*} u\right\rangle=0, \quad \text { for all } u \in H
$$

and

$$
P^{*} u \in \mathcal{K}^{*},
$$

where $K^{*}$ is the dual cone associated to $\mathcal{K}$. We have analogous results involving $Q,-\mathcal{K}$, and $(-\mathcal{K})^{*}$.

In order to prove the invariance of $\mathcal{K}$ and $-\mathcal{K}$, as we will see, it will be necessary to prove that $\mathcal{K}^{*} \subset-\mathcal{K}$ and $(-\mathcal{K})^{*} \subset \mathcal{K}$. In the classical argument developed by Weth in [14], the maximum principle to the operator $\Delta^{2}$ under certain boundary conditions and in certain domains is absolutely useful. Since this is not an option for us, let us prove some result that in some sense will substitute the lack of this result.

Lemma 2.10 For each radially symmetric $h \in C_{0}^{\infty}\left(\mathbb{R}^{N}\right), h \geq 0$, and $h \not \equiv 0$, there exists a positive continuous radial solution $v \in H_{\mathrm{rad}}^{2}\left(\mathbb{R}^{N}\right)$ of the linear problem

$$
\Delta^{2} v+V(x) v=h(x) \quad \text { in } \mathbb{R}^{N} .
$$


Proof The existence of a solution $v \in H_{\text {rad }}^{2}\left(\mathbb{R}^{N}\right)$ follows straightforwardly just by applying the Riesz theorem. Regularity is a simple matter just by calling Proposition 2.5 in [21].

To the positiveness we apply some arguments of Chabrowski and Yang in [15] which we describe below.

Since $v$ is smooth, $\Omega=\left\{x \in \mathbb{R}^{N} ; v(x)<0\right\}$ is an open subset of $\mathbb{R}^{N}$. Supposing by contradiction that there exists $x_{0} \in \Omega$, let $R>0$ such that

$$
B_{2 R}\left(x_{0}\right) \subset \Omega
$$

Assuming for simplicity that $R=1$, let us denote

$$
\bar{v}(x)= \begin{cases}v(x), & \text { if }\left|x-x_{0}\right| \leq 1, \\ 0, & \text { if }\left|x-x_{0}\right|>1,\end{cases}
$$

and $\rho \in C_{0}^{\infty}\left(\mathbb{R}^{N}\right)$ with $\operatorname{supp}(\rho) \subset B_{1}(0)$ and $\int \rho=1$. Let us define

$$
\tilde{v}(x)=\int r^{-N} \rho\left(\frac{x-y}{r}\right) \bar{v}(y) d y=\int_{B_{1}\left(x_{0}\right)} r^{-N} \rho\left(\frac{x-y}{r}\right) v(y) d y,
$$

where $0<r \leq 1$.

Note that $\operatorname{supp}(\tilde{v}) \subset B_{r+1}\left(x_{0}\right)$. Then, for $x \in B_{r+1}\left(x_{0}\right), \tilde{v}$ satisfies

$$
\begin{aligned}
\Delta^{2} \tilde{v}(x) & =\int_{B_{1}\left(x_{0}\right)} r^{-N} \Delta_{x}^{2} \rho\left(\frac{x-y}{r}\right) v(y) d y \\
& =\int_{B_{1}\left(x_{0}\right)} r^{-N-4} \rho\left(\frac{x-y}{r}\right)(h(y)-V(y) v(y)) d y=: \bar{h}(x),
\end{aligned}
$$

where $\bar{h} \geq 0$ as for each $y \in B_{r+1}(0), h(y)-V(y) v(y) \geq 0$. Then $\tilde{v}$ satisfies the following problem:

$$
\begin{cases}\Delta^{2} \tilde{v}=\bar{h} & \text { in } B_{r+1}(0) \\ \tilde{v}=\Delta \tilde{v}=0 & \text { on } \partial B_{r+1}(0)\end{cases}
$$

Let us recall that from standard minimization arguments and elliptic regularity theory, as proved in [15][Lemma 3], for all bounded smooth domain $\Omega \subset \mathbb{R}^{N}$ and for all $g \in L^{2 N /(N-4)}(\Omega) \cap C^{0, \alpha}(\Omega), 0<\alpha<1$, there exists a classical solution $w>0$ of

$$
\begin{cases}\Delta^{2} w=g & \text { in } \Omega, \\ w=\Delta w=0 & \text { on } \partial \Omega .\end{cases}
$$

For any $L^{2 N /(N-4)}\left(B_{r+1}\left(x_{0}\right)\right) \cap C^{0, \alpha}\left(B_{r+1}\left(x_{0}\right)\right)$ positive function $g$, let $w$ be the positive smooth solution of (2.10) with $\Omega=B_{r+1}\left(x_{0}\right)$. Using $w$ as a test function in (2.9) we obtain

$$
\begin{aligned}
\int_{B_{r+1}\left(x_{0}\right)} \bar{h} w d x & =\int_{B_{r+1}\left(x_{0}\right)} \Delta^{2} \tilde{v} w d x \\
& =\int_{B_{r+1}\left(x_{0}\right)} \tilde{v} \Delta^{2} w d x \\
& =\int_{B_{r+1}\left(x_{0}\right)} \tilde{v} g d x .
\end{aligned}
$$


However, while the last integral is negative, the first one is positive, which gives us a contradiction.

Using the last lemma it is possible to prove the following claim.

Claim $\mathcal{K}^{*} \subset-\mathcal{K}$.

Proof Let $u \in \mathcal{K}^{*}$. For each $h \in L^{2}\left(\mathbb{R}^{N}\right), h \geq 0$ a.e. in $\mathbb{R}^{N}$ let $\left(h_{n}\right)$ in $C_{0}^{\infty}\left(\mathbb{R}^{N}\right), h_{n} \geq 0$, such that $h_{n} \rightarrow h$ in $L^{2}\left(\mathbb{R}^{N}\right)$. For each $n \in \mathbb{N}$, let $v_{n}$ be the positive solution of the linear problem (2.8) with $h=h_{n}$, given by Lemma 2.10. Then $v_{n} \in \mathcal{K}$ and

$$
0 \geq\left\langle u, v_{n}\right\rangle=\int\left(\Delta u \Delta v_{n}+V(x) u v_{n}\right)=\int u h_{n} .
$$

Hence, by (2.11) and Fatou's lemma,

$$
\int u h \leq \liminf _{n \rightarrow \infty} \int u(x) h_{n}(x) \leq 0 .
$$

Then it follows that $u \leq 0$ a.e. in $\mathbb{R}^{N}$ and therefore $u \in-\mathcal{K}$.

Remark 2.11 It is worth pointing out that if $u \in H$, then $u=P u+P^{*} u$ where $P u \geq 0$ and $P^{*} u \leq 0$ a.e. in $\mathbb{R}^{N}$. Then $u \leq P u$, and consequently, $u^{+} \leq P u$ a.e. in $\mathbb{R}^{N}$. In the same way one can prove that $P^{*} u \leq u^{-}, Q u \leq u^{-}$, and $u^{+} \leq Q^{*} u$ a.e. in $\mathbb{R}^{N}$.

The following is a very important result to prove the invariance of $\mathcal{K}$ and $-\mathcal{K}$ under the flow $\varphi$.

Lemma 2.12 The operator $A$ satisfies the following conditions:

(i) $\langle A(u), v\rangle \leq\left\langle A\left(P^{*} u\right), v\right\rangle$, for all $u \in H$ and $v \in \mathcal{K}^{*}$;

(ii) $\langle A(u), v\rangle \leq\left\langle A\left(Q^{*} u\right), v\right\rangle$, for all $u \in H$ and $v \in(-\mathcal{K})^{*}$.

Proof Since (ii) can be proved in the same way, we just prove (i). Let $u \in H$ and $v \in \mathcal{K}^{*}$. By Remark 2.11, $P^{*} u \leq u^{-}$, and by $\left(\mathrm{f}_{5}\right)$

$$
f\left(x, P^{*} u(x)\right) \leq f\left(x, u^{-}(x)\right)
$$

Since $v \leq 0$, once more by $\left(\mathrm{f}_{5}\right)$ it follows that

$$
\begin{aligned}
\langle A(u), v\rangle & =\int f(x, u) v \\
& \leq \int f\left(x, u^{-}\right) v \\
& \leq \int f\left(x, P^{*} u\right) v=\left\langle A\left(P^{*} u\right), v\right\rangle .
\end{aligned}
$$

\section{Lemma 2.13}

(i) $A(\mathcal{K}) \subset \mathcal{K}$ and $A(-\mathcal{K}) \subset-\mathcal{K}$.

(ii) For sufficiently small $\alpha>0$, the $\alpha$-neighborhood of $\mathcal{K}, B_{\alpha}(\mathcal{K})$ is positively invariant under $\varphi$. Moreover, all critical points of I in $\overline{B_{\alpha}(\mathcal{K})}$ belong to $\mathcal{K}$. The same holds for the cone $-\mathcal{K}$. 
(iii) $\mathcal{K}$ and $-\mathcal{K}$ are positively invariant under $\varphi$.

Proof Let us prove the results just for $\mathcal{K}$, since for $-\mathcal{K}$ the arguments are the same.

(i) By Lemma 2.12(i), for $u \in \mathcal{K}$,

$$
\begin{aligned}
\left\|P^{*}(A(u))\right\|^{2} & =\langle A(u)-P(A(u)), A(u)-P(A(u))\rangle \\
& =\left\langle A(u), P^{*}(A(u))\right\rangle \\
& \leq\left\langle A\left(P^{*} u\right), P^{*}(A(u))\right\rangle=0,
\end{aligned}
$$

which implies that $A(u) \in \mathcal{K}$.

(ii) Let $u \in H$, by Lemma 2.3 and Lemma 2.12(i), for $0<\epsilon<1$, we have

$$
\begin{aligned}
\left\|P^{*}(A(u))\right\|^{2} & =\left\langle A(u), P^{*}(A(u))\right\rangle \\
& \leq\left\langle A\left(P^{*} u\right), P^{*}(A(u))\right\rangle \\
& \leq\left\|P^{*}(A(u))\right\|\left(\epsilon\left\|P^{*} u\right\|+A(\epsilon)\left\|P^{*} u\right\|^{p+1}\right),
\end{aligned}
$$

which implies that

$$
\left\|P^{*}(A(u))\right\| \leq \epsilon\left\|P^{*} u\right\|+A(\epsilon)\left\|P^{*} u\right\|^{p+1}=\left\|P^{*} u\right\|\left(\epsilon+A(\epsilon)\left\|P^{*} u\right\|^{p}\right),
$$

since $\left\|P^{*}(A(u))\right\| \neq 0$. Then, if $0<\left\|P^{*} u\right\|<\left(\frac{1-\epsilon}{2 A(\epsilon)}\right)^{\frac{1}{p}}=: \alpha_{0}$,

$$
\left\|P^{*}(A(u))\right\|<\left\|P^{*} u\right\|
$$

Hence, for all $\alpha<\alpha_{0}$, every fixed point of $A$ in $\overline{B_{\alpha}(\mathcal{K})}$ belongs to $\mathcal{K}$. In fact, if $u \in \overline{B_{\alpha}(\mathcal{K})} \backslash \mathcal{K}$ and $A(u)=u$ then $0<\left\|P^{*} u\right\| \leq \alpha<\alpha_{0}$, which implies by (2.13) that

$$
\left\|P^{*} u\right\|=\left\|P^{*}(A(u))\right\|<\left\|P^{*} u\right\|
$$

which is a contradiction.

Now let us prove that $B_{\alpha}(\mathcal{K})$ is positively invariant.

Note that by (2.13)

$$
A\left(\partial B_{\alpha}(\mathcal{K})\right) \subset \operatorname{int}\left(B_{\alpha}(\mathcal{K})\right)
$$

Suppose, contrary to our claim, that there exists $u_{0} \in B_{\alpha}(\mathcal{K})$ such that $\varphi\left(t_{0}, u_{0}\right) \in \partial B_{\alpha}(\mathcal{K})$ where $t_{0} \in\left[0, T\left(u_{0}\right)\right)$ is the least positive real with this property. As $B_{\alpha}(\mathcal{K})$ is a convex open set and $\left\{\varphi\left(t_{0}, u_{0}\right)\right\}$ is compact, by Mazur's separation theorem, there exist a linear functional $\rho \in H^{*}$ and a real number $\beta$ such that $\rho\left(\varphi\left(t_{0}, u_{0}\right)\right)=\beta$ and $\rho(u)>\beta$ for all $u \in B_{\alpha}(\mathcal{K})$.

By (2.14) it follows that

$$
\begin{aligned}
\left.\frac{\partial}{\partial t} \rho\left(\varphi\left(t, u_{0}\right)\right)\right|_{t=t_{0}} & =\rho\left(-\nabla I\left(\varphi\left(t_{0}, u_{0}\right)\right)\right) \\
& =\rho\left(A\left(\varphi\left(t_{0}, u_{0}\right)\right)\right)-\beta>0 .
\end{aligned}
$$


Therefore, there exists $\eta>0$ such that $\rho\left(\varphi\left(t, u_{0}\right)\right)<\beta$ as long as $t \in\left(t_{0}-\eta, t_{0}\right)$. Then $\varphi\left(t, u_{0}\right) \notin B_{\alpha}(\mathcal{K})$ for all $t \in\left(t_{0}-\eta, t_{0}\right)$, which contradicts the minimality of $t_{0}$.

(iii) This item follows straightforwardly observing that $\mathcal{K}=\bigcap_{\alpha>0} B_{\alpha}(\mathcal{K})$.

From now on let us consider $\alpha>0$ such that the statement of Lemma 2.13 holds for $\mathcal{K}$ and $-\mathcal{K}$.

To obtain the signed solutions we will use the following result.

Proposition 2.14 Assume that there exists $u_{0} \in \mathcal{K}$ such that $I\left(u_{0}\right)<0$, then there exists a nontrivial critical point of $I$ in $\mathcal{K}$. The same holds for $-\mathcal{K}$.

Proof First note that by definition of $\mathcal{A}_{0}, I(u) \geq 0$ for all $u \in \mathcal{A}_{0}$. Then by continuity, $I(u) \geq$ 0 for all $u \in \overline{\mathcal{A}}_{0}$.

Since $I\left(u_{0}\right)<0$ then $u_{0} \notin \overline{\mathcal{A}}_{0}$. As $\mathcal{A}_{0}$ is an open neighborhood of the origin, there exists $s \in(0,1)$ such that $s u_{0} \in \partial \mathcal{A}_{0} \cap \mathcal{K}$. Since $\partial \mathcal{A}_{0} \cap \mathcal{K}$ is a closed positively invariant set, by Proposition 2.4, $\omega\left(s u_{0}\right) \subset \partial \mathcal{A}_{0} \cap \mathcal{K}$ is non-empty and any of its points are critical points of $I$.

Let us denote by

$$
\mathcal{A}_{+}=\mathcal{A}\left(B_{\alpha}(\mathcal{K})\right) \cap \partial \mathcal{A}_{0} \quad \text { and } \quad \mathcal{A}_{-}=\mathcal{A}\left(B_{\alpha}(-\mathcal{K})\right) \cap \partial \mathcal{A}_{0} .
$$

Lemma 2.15 $\mathcal{A}_{+}$and $\mathcal{A}_{-}$are disjoint relatively open sets of $\partial \mathcal{A}_{0}$.

Proof Since $B_{\alpha}(\mathcal{K})$ and $B_{\alpha}(-\mathcal{K})$ are open sets, then so are $\mathcal{A}\left(B_{\alpha}(\mathcal{K})\right)$ and $\mathcal{A}\left(B_{\alpha}(-\mathcal{K})\right)$.

Suppose, contrary to our claim, that there exists $u \in \mathcal{A}_{+} \cap \mathcal{A}_{-}$. Since $u \in \partial \mathcal{A}_{0}$, then $T(u)=$ $\infty$ and $\omega(u) \neq \emptyset$. Further, since $u \in \mathcal{A}\left(B_{\alpha}(\mathcal{K})\right) \cap \mathcal{A}\left(B_{\alpha}(-\mathcal{K})\right)$, then $\omega(u) \subset \overline{B_{\alpha}(\mathcal{K})} \cap \overline{B_{\alpha}(-\mathcal{K})}$. But since $\omega(u)$ consists of critical points of $I$, by Lemma 2.13, $\omega(u) \in \mathcal{K} \cap-\mathcal{K}=\{0\}$, which contradicts the fact that $u \in \partial \mathcal{A}_{0}$.

Proposition 2.16 Suppose that there exists a continuous path $h:[0,1] \rightarrow H$, such that $h(0) \in \mathcal{K}, h(1) \in-\mathcal{K}$, and $I(h(t))<0$ for all $t \in[0,1]$. Then I has at least three nontrivial critical points, these being $u_{1} \in \mathcal{K}, u_{2} \in-\mathcal{K}$, and $u_{3} \in H \backslash(\mathcal{K} \cup-\mathcal{K})$.

Proof Proposition 2.14 gives the existence of the signed critical points $u_{1} \in \mathcal{K}$ and $u_{2} \in-\mathcal{K}$.

To get the nodal one, let us first highlight that $h([0,1]) \cap \overline{\mathcal{A}}_{0}=\emptyset$.

Let $Q=[0,1]^{2}$ and $\mathcal{B} \subset Q$ be defined by

$$
\mathcal{B}=\left\{\left(s_{1}, s_{2}\right) \in Q ; s_{1} h\left(s_{2}\right) \in \mathcal{A}_{0}\right\} .
$$

Note that $B$ is relatively open in $Q$ and the following hold:

- $\{0\} \times[0,1] \subset \mathcal{B}$, since $0 h(s)=0 \in \mathcal{A}_{0}, \forall s \in[0,1]$;

- $\{1\} \times[0,1] \cap \overline{\mathcal{B}}=\emptyset$, since $I(h(s))<0, \forall s \in[0,1]$.

By recalling the Leray-Schauder continuation principle, it follows that there exists a connected component $\Gamma$ of $\partial \mathcal{B}$, such that

$$
\Gamma \cap([0,1] \times\{0\}) \neq \emptyset \quad \text { and } \quad \Gamma \cap([0,1] \times\{1\}) \neq \emptyset .
$$


Now, let us consider $\Sigma$ the closure of the connected component of $\Gamma \backslash \partial Q$ that intersects $[0,1] \times\{0\}$ and $[0,1] \times\{1\}$.

Denoting by $\Gamma_{0}=\left\{s_{1} h\left(s_{2}\right) ;\left(s_{1}, s_{2}\right) \in \Sigma\right\}$, we find that $\Gamma_{0}$ is a connected subset of $\partial \mathcal{A}_{0}$ such that $\Gamma_{0} \cap \pm \mathcal{K} \neq \emptyset$. Since by Lemma 2.15, $\mathcal{A}_{ \pm}$are disjoint open subsets of $\partial \mathcal{A}_{0}, \Gamma_{0} \cap \mathcal{A}_{ \pm}$ are open disjoints subsets of $\Gamma_{0}$. By connectedness of $\Gamma_{0}$, there exists $u \in \Gamma_{0} \backslash\left(\mathcal{A}_{+} \cup \mathcal{A}_{-}\right)$ and once $\partial \mathcal{A}_{0} \backslash\left(\mathcal{A}_{+} \cup \mathcal{A}_{-}\right)$is positively invariant, then $\{\varphi(t, u) ; t \geq 0\} \subset \partial \mathcal{A}_{0} \backslash\left(\mathcal{A}_{+} \cup \mathcal{A}_{-}\right)$. Using the fact that this is a closed subset in $\partial \mathcal{A}_{0}$, it follows that $\omega(u) \subset \partial \mathcal{A}_{0} \backslash\left(\mathcal{A}_{+} \cup \mathcal{A}_{-}\right)$. In particular, $\omega(u) \cap(\mathcal{K} \cup-\mathcal{K}) \neq \emptyset$ and any of his points are nodal critical points of $I$.

The next result will be useful to put the energy functional $I$ in the context of the last proposition.

Lemma 2.17 If $S \subset H \backslash\{0\}$ is a compact subset and $\tilde{S}=\{t u ; u \in S$ and $t \geq 0\}$, then

$$
I(u) \rightarrow-\infty \quad \text { as } u \in \tilde{S} \text { and }\|u\| \rightarrow \infty .
$$

Proof Let $\left(u_{n}\right) \subset \tilde{S}$ be a sequence such that $\left\|u_{n}\right\| \rightarrow \infty$. Then there exists a sequence in $\mathbb{R}_{+}\left(t_{n}\right)$ such that $u_{n}=t_{n} v_{n}$ and $\left(v_{n}\right) \subset S$. Since $S$ is compact, we can suppose that along a subsequence $v_{n} \rightarrow v$, as $n \rightarrow \infty$, for some $v \in S$. As $\left\|u_{n}\right\| \rightarrow \infty$, then one trivially sees that $t_{n} \rightarrow \infty$.

Now let us prove that

$$
\lim _{n \rightarrow \infty} \int \frac{F\left(x, t_{n} v_{n}\right)}{t_{n}^{2}}=+\infty
$$

In fact, let $\Gamma=\left\{x \in \mathbb{R}^{N} ; v(x) \neq 0\right\}$, then by Fatou's lemma and $\left(\mathrm{f}_{4}\right)$, it follows that

$$
\begin{aligned}
\lim _{n \rightarrow \infty} \int \frac{F\left(x, t_{n} v_{n}\right)}{t_{n}^{2}} & =\lim _{n \rightarrow \infty} \int \frac{F\left(x, t_{n} v_{n}\right)}{t_{n}^{2} v_{n}^{2}} v_{n}^{2} \\
& \geq \lim _{n \rightarrow \infty} \int_{\Gamma} \frac{F\left(x, t_{n} v_{n}\right)}{t_{n}^{2} v_{n}^{2}} v_{n}^{2}=+\infty
\end{aligned}
$$

Let $M>0$ be such that $\left\|v_{n}\right\| \leq M$, for all $n \in \mathbb{N}$. Then we have

$$
\begin{aligned}
I\left(u_{n}\right) & =I\left(t_{n} v_{n}\right) \\
& =t_{n}^{2}\left(\frac{\left\|v_{n}\right\|^{2}}{2}-\int \frac{F\left(x, t_{n} v_{n}\right)}{t_{n}^{2}}\right) \\
& \leq t_{n}^{2}\left(\frac{M^{2}}{2}-\int \frac{F\left(x, t_{n} v_{n}\right)}{t_{n}^{2}}\right) \\
& \rightarrow-\infty
\end{aligned}
$$

as $n \rightarrow \infty$.

Now, let us prove the main result of this work

Proof of Theorem 1.3 Let $u \in \mathcal{K}$ and $v \in \mathcal{K}^{*}$, such that $u, v \neq 0$ and $u$ and $v$ are linearly independent in $H$. For each $s>0$, let us define $h_{s}:[0,1] \rightarrow H$ as $h_{s}(t)=s(t u+(1-t) v)$. 
For each $s>0$,

- $h_{s}(1)=s u \in \mathcal{K} \backslash\{0\}$,

- $h_{s}(0)=s v \in \mathcal{K}^{*} \backslash\{0\}$.

By Lemma 2.17 applied to the compact set $S=\{t u+(1-t) v ; t \in[0,1]\}$, we see that if $s$ is large enough, then $I\left(h_{s}(t)\right)<0, \forall t \in[0,1]$. Hence, Proposition 2.16 gives us the existence of $u_{1}, u_{2}$, and $u_{3}$, respectively, a positive, a negative, and a nodal critical point of $I$ restricted to $H$. The existence of the critical points of $I$ in all space $H^{2}\left(\mathbb{R}^{N}\right)$ follows just by applying the principle of symmetric criticality of Palais to the functional $I$, once we have observed that $I$ is invariant by the action of the group $O(N)$, and

$$
H_{\mathrm{rad}}^{2}\left(\mathbb{R}^{N}\right)=\left\{u \in H^{2}\left(\mathbb{R}^{N}\right) ; u(g(x))=u(x), \forall g \in O(N)\right\} .
$$

Hence the theorem follows.

\section{Competing interests}

The author declares that he has no competing interests.

\section{Author's contributions}

The author wrote and approved the final manuscript.

\section{Acknowledgements}

The author would like to thank Prope-Unesp and Fundunesp for the support and Prof. Sérgio Monari Soares and Antonio Suárez for useful suggestions concerning the paper. This work was supported by FAPESP - 2012/20160-0 - 2014/16136-1 and CNPq 442520/2014-0

Received: 20 August 2014 Accepted: 4 January 2015 Published online: 31 January 2015

\section{References}

1. Floer, A, Weinstein, A: Nonspreading wave packets for the cubic Schrödinger equations with a bounded potential. J. Funct. Anal. 69, 397-408 (1986)

2. Rabinowitz, PH: On a class of nonlinear Schrödinger equations. Z. Angew. Math. Phys. 43, $270-291$ (1992)

3. Wang, X: On concentration of positive bound states of nonlinear Schrödinger equations. Commun. Math. Phys. 153, 229-244 (1993)

4. del Pino, M, Felmer, P: Local mountain pass for semilinear elliptic problems in unbounded domains. Calc. Var. Partial Differ. Equ. 4, 121-137 (1996)

5. Bonheure, D, Schaftingen, JV: Bound state solutions for a class of nonlinear Schrödinger equations. Rev. Mat. Iberoam. 24, 297-351 (2008)

6. Bonheure, D, Di Cosmo, J, Schaftingen, JV: Nonlinear Schrödinger equation with unbounded or vanishing potentials: solutions concentrating on lower dimensional spheres. J. Differ. Equ. 252, 941-968 (2012)

7. Pimenta, MTO, Soares, SHM: Existence and concentration of solutions for a class of biharmonic equations. J. Math. Anal. Appl. 390, 274-289 (2012)

8. Pimenta, MTO, Soares, SHM: Singularly perturbed biharmonic problems with superlinear nonlinearities. Adv. Differ. Equ. 19, 31-50 (2014)

9. Figueiredo, GJM, Pimenta, MTO: Multiplicity of solutions for a biharmonic equation with subcritical or critical growth. Bull. Belg. Math. Soc. Simon Stevin 20(3), 519-534 (2013)

10. Castro, A, Cossio, J, Neuberger, J: A sign-changing solution for a superlinear Dirichlet problem. Rocky Mt. J. Math. 27(4), 1041-1053 (1997)

11. Alves, CO, Soares, SHM: On the location and profile of spyke-layer nodal solutions to nonlinear Schrödinger equations. J. Math. Anal. Appl. 296, 563-577 (2004)

12. Alves, CO, Soares, SHM: Nodal solutions for singularly perturbed equations with critical exponential growth. J. Differ. Equ. 234, 464-484 (2007)

13. Moreau, JJ: Décomposition orthogonale d'un espace hilbertien selon deux cônes mutuellement polaires. C. R. Math. Acad. Sci. Paris 255, 238-240 (1962)

14. Weth, T: Nodal solutions to superlinear biharmonic equations via decomposition in dual cones. Topol. Methods Nonlinear Anal. 28, 33-52 (2006)

15. Chabrowski, J, Yang, J: Nonnegative solutions for semilinear biharmonic equations in $\mathbb{R}^{N}$. Analysis 27, 35-59 (1997)

16. Ebihara, Y, Schonbek, T: On the (non)compactness of the radial Sobolev spaces. Hiroshima Math. J. 16, 665-669 (1986)

17. Huang, Y, Liu, X: On sing-changing solution for a fourth-order asymptotically linear elliptic problem. Nonlinear Anal. 72, 2271-2276 (2010)

18. Huang, Y, Liu, X: Sign-changing solutions for $p$-biharmonic equations with Hardy potential. J. Math. Anal. Appl. 412, 142-154 (2014)

19. Liu, Z, Sun, J: Invariant sets of descending flow in critical point theory with applications to nonlinear differential equations. J. Differ. Equ. 172, 257-299 (2001) 
20. Miyagaki, O, Souto, M: Superlinear problems without Ambrosetti and Rabinowitz growth condition. J. Differ. Equ. 245, 3628-3638 (2008)

21. Ramos, M: Uniform estimates for the biharmonic operator in $\mathbb{R}^{N}$ and applications. Commun. Appl. Anal. 8, 435-457 (2009)

Submit your manuscript to a SpringerOpen ${ }^{\circ}$ journal and benefit from:

- Convenient online submission

- Rigorous peer review

- Immediate publication on acceptance

- Open access: articles freely available online

- High visibility within the field

- Retaining the copyright to your article

Submit your next manuscript at $>$ springeropen.com 